\title{
ERG and FLI1 protein expression in epithelioid sarcoma
}

\author{
David L Stockman ${ }^{1}$, Jason L Hornick ${ }^{2}$, Michael T Deavers ${ }^{1}$, Dina C Lev ${ }^{3,4}$, \\ Alexander J Lazar ${ }^{1,4}$ and Wei-Lien Wang ${ }^{1}$ \\ ${ }^{1}$ Departments of Pathology, University of Texas M.D. Anderson Cancer Center, Houston, TX, USA; \\ ${ }^{2}$ Department of Pathology, Brigham and Women's Hospital and Harvard Medical School, Boston, MA, USA; \\ ${ }^{3}$ Department of Cancer Biology, University of Texas M.D. Anderson Cancer Center, Houston, TX, USA and \\ ${ }^{4}$ Sarcoma Research Center, University of Texas M.D. Anderson Cancer Center, Houston, TX, USA
}

\begin{abstract}
Epithelioid sarcoma is a rare, aggressive keratin-positive sarcoma that co-expresses CD34 in $50 \%$ of cases and may mimic an angiosarcoma. Recently, we have observed one case of epithelioid sarcoma that labeled for ERG, an ETS family regulatory transcription factor, which is considered to be a reliable marker for vascular differentiation. We investigated the prevalence of nuclear expression of ERG and FLI1, a homologous transcription factor, in these tumors. A formalin-fixed paraffin-embedded tissue microarray of 37 epithelioid sarcomas was examined. Immunohistochemistry was performed using anti-ERG monoclonal antibody to the $\mathrm{N}$-terminus, anti-ERG monoclonal antibody to the C-terminus and anti-FLI1 monoclonal antibody. Comparison was made with CD34, CD31, and D2-40 labeling. The extent of immunoreactivity was graded according to the percentage of positive tumor cell nuclei (0: no staining; $1+:<5 \% ; 2+: 5-25 \% ; 3+: 26-50 \% ; 4+: 51-75 \%$; and $5+: 76-100 \%$ ), and the intensity of staining was graded as weak, moderate, or strong. Nuclear staining for the $\mathrm{N}$-terminus of ERG was seen in 19 out of 28 cases: 10 with diffuse( 4 to $5+$ ) strong/moderate labeling; 1 with $2+$ moderate labeling and 8 with weak labeling ( 1 to $4+, 2$ each). Focal staining for the C-terminus of ERG was seen in only 1 out of 29 cases ( $2+$ moderate). FLI1 labeling was seen in nearly all (28 out of 30) cases: 16 with diffuse $(5+)$ predominantly moderate labeling, and 8 cases with diffuse $(5+)$ weak labeling. The remainder had variable moderate ( 1 to $3+$ ) or weak (1 to $4+$ ) FLI1 staining. CD34 was positive in 22 out of 30 cases and D2-40 was found to be positive in 22 out of 31 cases. All cases were negative for CD31 ( 0 out of 30). Epithelioid sarcoma can label with antibodies to the N-terminus of ERG, FLI1, and D2-40, which may cause diagnostic confusion for a vascular tumor. A panel of other antibodies including SMARCB1 and CD31 should be used in evaluating these tumors. ERG antibody selection is also critical, as those directed against the C-terminus are less likely to label epithelioid sarcoma.
\end{abstract}

Modern Pathology (2014) 27, 496-501; doi:10.1038/modpathol.2013.161; published online 27 September 2013

Keywords: epithelioid sarcoma; ERG; FLI1

ERG and FLI1, members of the ETS family of transcription factors, are markers of endothelial differentiation. The utility of ERG and FLI1 in distinguishing cutaneous and non-cutaneous angiosarcomas from other histologic mimics has been previously described. ${ }^{1-4}$ Recently, a recurrent epithelioid sarcoma at our institution showed histologic features suggestive of vascular differentiation: hemorrhagic at low power, discohesive sheets of large atypical epithelioid cells, and areas of

Correspondence: Dr Wei-Lien Wang, MD, Department of Pathology, University of Texas M.D. Anderson Cancer Center, 1515 Holcombe Boulevard Unit 085, Houston, TX 77030, USA. E-mail: wlwang@mdanderson.org

Received 17 May 2013; revised 1 July 2013; accepted 2 July 2013; published online 27 September 2013 hobnailed-appearing cells in blood-filled cystic spaces mimicking epithelioid angiosarcoma. (Figure 1) Immunohistochemical studies showed the tumor to be reactive for keratin, CD34, and ERG but negative for CD31; SMARCB1 expression was lost. Initial biopsies showed central geographic necrosis with palisading tumor cells, characteristic of many cases of epithelioid sarcoma (Figure 2).

The purpose of our study was to characterize the immunohistochemical expression of ERG and FLI1 in epithelioid sarcoma, as expression of these markers may lead to the misperception of vascular differentiation and a potential diagnostic pitfall. Comparison with labeling patterns of other markers associated with vascular differentiation, including CD34, D2-40, and CD31, was also made. 




Figure 1 Recurrent epithelioid sarcoma mimicking a vascular tumor. The tumor is composed of (a) hemorrhage and (b) epithelioid cells. (c) Diffuse reactivity for ERG was seen (using an antibody directed against the N-terminus).

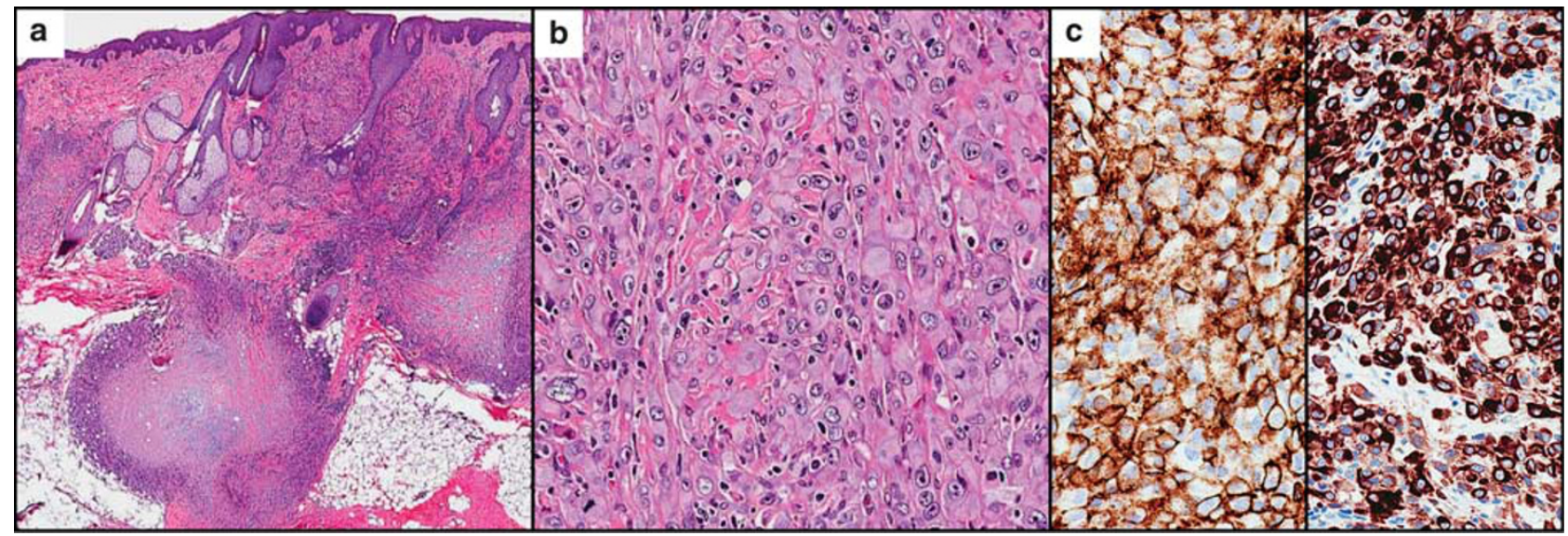

Figure 2 Initial biopsy demonstrating classic features of epithelioid sarcoma. (a) Multiple nodules with central necrosis involving the dermis and subcutis. (b) High-power examination reveals atypical epithelioid cells, which (c) co-express pankeratin (left) and CD34 (right). SMARCB1 expression was lost (not shown).

\section{Materials and methods}

With institutional review board approval, 37 cases of previously diagnosed epithelioid sarcoma were selected from the pathology files of the University of Texas M.D. Anderson Cancer Center. Inclusion criteria for all cases of epithelioid sarcomas included appropriate histological features, immunohistochemical evidence of keratin positivity and loss of nuclear expression of SMARCB1. Archived paraffin blocks from 37 localized epithelioid sarcomas and 8 primary breast cancer surgical samples (controls for epithelial-related markers) were assembled in a tissue microarray and processed as previously published. ${ }^{5}$

Immunohistochemical study was performed following pressure cooker antigen retrieval using a mouse anti-ERG monoclonal antibody to the N-terminus (1:2000; 9FY; BioCare Medical, Concord, CA, USA), a rabbit anti-ERG monoclonal antibody to the C-terminus (1:2000; EPR3864(2); Epitomics,
Burlingame, CA, USA), and a mouse anti-FLI1 monoclonal antibody (1:100; G146-222; BD Biosciences, San Jose, CA, USA). Comparison was made to mouse anti-CD34 monoclonal antibody (1:40; my10, BD Biosciences), mouse anti-CD31 monoclonal antibody (1:30, 1A10, NovoCastra Vision Biosystems, Newcastle upon Tyne, UK), and mouse anti-D2-40 monoclonal antibody (1:50; Covance, Princeton, NJ, USA). In evaluable cores, immunoreactivity extent was graded according to the percentage of positive tumor cell nuclei ( 0 : no staining; $1+:<5 \% ; 2+: 5-25 \% ; 3+: 26-50 \% ; 4+: 51-75 \%$; and $5+: 76-100 \%$ ) and the intensity of labeling was graded as weak, moderate, or strong.

\section{Results}

Immunohistochemical results are summarized in Table 1 and examples of positive immunohistochemical labeling shown in Figure 3. Distinct 
ERG FLl1 expression in epithelioid sarcoma

Table 1 Summary of immunohistochemical labeling patterns for ERG (N-terminus and C-terminus), FLI1, CD34, D2-40 and CD31

\begin{tabular}{|c|c|c|c|c|c|c|}
\hline & ERG (N-terminus) & ERG (C-terminus) & FLI1 & $C D 34$ & $D 2-40$ & CD31 \\
\hline$N$ & 28 & 29 & 30 & 30 & 31 & 30 \\
\hline Positive & $19(68 \%)$ & $1(3 \%)$ & $28(93 \%)$ & $22(73 \%)$ & $22(71 \%)$ & 0 \\
\hline \multicolumn{7}{|l|}{ Extent } \\
\hline $5+$ & $7(25 \%)$ & 0 & $24(80 \%)$ & $17(57 \%)$ & $21(67 \%)$ & 0 \\
\hline $4+$ & $5(18 \%)$ & 0 & $1(3 \%)$ & 0 & 0 & 0 \\
\hline $3+$ & $2(7 \%)$ & 0 & $1(3 \%)$ & 0 & 0 & 0 \\
\hline $2+$ & $3(11 \%)$ & $1(3 \%)$ & 0 & $4(13 \%)$ & 0 & 0 \\
\hline $1+$ & $2(7 \%)$ & 0 & $2(7 \%)$ & $1(3 \%)$ & $1(3 \%)$ & 0 \\
\hline 0 & $9(32 \%)$ & $28(97 \%)$ & $2(7 \%)$ & $8(27 \%)$ & $9(29 \%)$ & $30(100 \%)$ \\
\hline \multicolumn{7}{|l|}{ Degree } \\
\hline Strong & $6(21 \%)$ & 0 & $1(3 \%)$ & $13(43 \%)$ & $9(29 \%)$ & 0 \\
\hline Moderate & $5(18 \%)$ & $1(3 \%)$ & $17(57 \%)$ & $5(17 \%)$ & $7(23 \%)$ & 0 \\
\hline Weak & $8(29 \%)$ & 0 & $10(33 \%)$ & $4(13 \%)$ & $6(19 \%)$ & 0 \\
\hline None & $9(32 \%)$ & $28(97 \%)$ & $2(7 \%)$ & $8(27 \%)$ & $9(29 \%)$ & $30(100 \%)$ \\
\hline
\end{tabular}



Figure 3 Examples of (a) ERG, (b) FLI1, and (c) CD34 (5+, strong) and (d) D2-40 labeling (5 +, strong) in epithelioid sarcoma.

nuclear staining for the N-terminus of ERG was observed in 19 out of 28 cases (68\%) of epithelioid sarcoma $(43 \%$ strong, $4+$ or $5+$ and patchy moderate to weak staining in 7 out of 28 cases
$(25 \%))$. Only one case labeled with the antibody to the C-terminus of ERG with moderate patchy $(2+)$ labeling. FLI1 labeled almost all assessed epithelioid sarcoma cases (28 out of $30,93 \%$ ); 16 had 
Table 2 Summary of co-expression of markers (N-terminus ERG, CD34, D2-40)

\begin{tabular}{lc}
\hline & $\mathrm{N}=25(\%)^{\mathrm{a}}$ \\
\hline ERG/D2-40 & $5(20)$ \\
ERG/CD34 & $3(12)$ \\
D2-40/CD34 & $5(20)$ \\
All three & $7(28)$ \\
Only one & $5(20)$ \\
\hline
\end{tabular}

$\mathrm{a}_{25}$ out of 37 had evaluable cores in all three immunohistochemical studies.

diffuse $(5+)$ predominantly moderate labeling, and 8 cases showed diffuse $(5+)$ weak labeling. The remainder had variable moderate $(1$ to $3+)$ or weak (1 to $4+$ ) FLI1 staining. CD34 was positive in 22 out of 30 cases $(73 \%)$ with strong $4+$ or $5+$ staining in $57 \%$ of cases. D2-40 was positive in 22 out of 31 cases $(71 \%)$ with the majority $(n=15)$ having moderate to strong diffuse $5+$ labeling. All cases (0/30) were negative for CD31. Twenty out of 25 cases had labeling with at least two antibodies (either N-terminus to ERG, FLI1 and D2-40), with seven cases labeling for all three markers. Table 2 summarizes co-expression labeling patterns.

\section{Discussion}

We found that epithelioid sarcoma can be reactive with antibodies directed against ERG (N-terminus) and FLI1. Prior studies have examined the specificity of ERG for vascular differentiation, although only small numbers of epithelioid sarcoma were tested before. Miettinen et al found that a monoclonal antibody to the N-terminus of ERG (CPDR ERG) was sensitive and specific for vascular differentiation in a large series of vascular tumors $(n=250) .{ }^{3}$ ERG was expressed in all hemangiomas and lymphangiomas, 96 of 100 angiosarcomas, 42 of 43 epithelioid hemangioendotheliomas, and all 26 Kaposi sarcomas. Among the nonvascular tumors, only blastic extramedullary myeloid tumors $(7 / 10$, $70 \%)$ and rare cases of Ewing sarcoma (2/29, 7\%) were positive. Eight epithelioid sarcomas were also tested; none had any reactivity, in contrast to our series wherein $67 \%$ of cases had labeling. Although not certain, difference in technique and small sample size may partially explain the discrepancy. Yaskiv et $a l^{4}$ also found ERG to be a reliable marker for vascular differentiation with all 125 vascular proliferations labeling with an ERG antibody to the C-terminus of ERG (EPR3864, Epitomics). A limited number of non-vascular mesenchymal tumor types were also tested; only six of eight meningiomas were also found to express ERG. Epithelioid sarcomas were not tested. Using the same antibody, ERG and FLI1 expression were found to be sensitive and specific for angiosarcoma in a large series of cutaneous tumors $(n=72){ }^{1}$ All 23 angiosarcomas (vasoformative/angiomatoid, spindled, and epithelioid) demonstrated strong and extensive labeling patterns, whereas the remaining tested tumor types (melanoma, squamous cell carcinoma, atypical fibroxanthoma, leiomyosarcoma) were negative. In contrast, FLI1 (same antibody used as in our study) was found to be less specific, labeling $87 \%(13 / 15)$ of squamous cell carcinomas, 59\% (10 out of 17) of melanomas, $92 \%$ (11 out of 12) of atypical fibroxanthomas and $20 \%$ ( 1 out of 5 ) of leiomyosarcomas. Epithelioid sarcoma was also not examined in that study. Folpe et $a l^{2}$ found antibodies to FLI1 (Sc 356-polyclonal, Santa Cruz Biotechnology, Santa Cruz, CA, USA) to be highly sensitive for vascular differentiation, labeling 50 out of $53(93 \%)$ various vascular tumors while being negative in 16 non-vascular mesenchymal tumors including 6 epithelioid sarcomas. ${ }^{3}$ One possible explanation for the differences seen in this prior and our study is the different antibody used.

Our series adds to the understanding of ERG and FLI1 expression, particularly in cutaneous tumors, by demonstrating the majority of epithelioid sarcomas are reactive for the N-terminus of ERG (19 out of $28,68 \%$ ) and FLI1 (28 out of $30,93 \%$ ). Awareness of this expression pattern is important, as the histological spectrum of epithelioid sarcoma can sometimes overlap with a vascular tumor with irregular cystic spaces and clefts, loss of cellular cohesion, angiomatoid areas found near the periphery of the lesion, and hemorrhage. ${ }^{4,6,7}$ Epithelioid sarcoma can even show cytoplasmic vacuolization without true lumen formation, ${ }^{8}$ leading to diagnostic confusion with an epithelioid hemangioendothelioma ${ }^{8-10}$ and epithelioid angiosarcoma. ${ }^{11}$ Furthermore, epithelioid sarcoma characteristically co-expresses keratin $(75 \%)^{12}$ and CD34 $\left.(50 \%)\right)^{13}$ which together with expression of ERG can closely mimic the immunophenotype of angiosarcoma. In our series, $80 \%$ (20 out of 25) of cases co-expressed at least D2-40, ERG, or CD34 with 28\% (7 out of 25) expressing of all three markers.

Fortunately, epithelioid sarcoma generally exhibits loss of SMARCB1 expression in contrast to angiosarcomas. SMARCB1 loss is considered highly sensitive $(93 \%)^{14}$ and variably specific for epithelioid sarcoma. ${ }^{15}$ Disruption of the SMARCB1 gene on 22q11 resulting in loss of protein expression by immunohistochemistry can occur by a variety of mechanisms in epithelioid sarcoma, including homozygous deletion ${ }^{16}$ or rearrangements. ${ }^{15}$ All of our cases had loss of SMARCB1 and an absence of CD31 expression. In addition, an antibody to the C-terminus of ERG was much less likely to react with epithelioid sarcomas, in contrast to an antibody directed against the $\mathrm{N}$-terminus.

$E R G$, located on $21 \mathrm{q} 22$, and $F L I$, located on $11 \mathrm{q} 21$, are members of the ETS family and have a highly conserved 85 amino-acid winged helix-loop-helix 
domain, which mediates binding to DNA with GGAA/T-containing sequences. ${ }^{17}$ Both are involved in hematopoietic and endothelial development. Previous studies have shown that some epithelioid sarcomas have amplifications and losses involving chromosomes 3, 4, 18, 9, and 11 with copy number gains including 11q13 seen in five cases, one of which was angiomatoid. Lushnikova et al also found cyclin D1, located in 11q13, to be overexpressed in 9 of 15 epithelioid sarcomas, some of which, but not all, correlated to those with gains in 11q13. ${ }^{18}$ Given the proximity of FLI1, perhaps a similar phenomenon is occurring in 11q21 with some epithelioid sarcomas having copy gains resulting in overexpression of FLI1. It is interesting that antibodies to the C-terminus of ERG were less likely to label epithelioid sarcoma than the N-terminus. In a previous study, an antibody to the C-terminus of ERG was found to be more specific for ERG expression in Ewing sarcoma harboring a EWSR1-ERG fusion transcript than an antibody to the N-terminus. ${ }^{19}$ ERG and FLI1 proteins share $68 \%$ of amino-acid sequences overall and $98 \%$ of the ETS-binding domain. Therefore, it is possible that in some cases, the antibodies to the N-terminus of ERG may recognize similar epitopes as the antibodies to FLI1, although not all of our FLI1-positive cases labeled for the N-terminus of ERG. FLI1 and ERG are overexpressed secondary to rearrangement in various tumor types including prostate carcinomas, Ewing sarcoma, and acute myeloid leukemia, which has been implicated in tumorigenesis. ${ }^{3,19,20}$ The role of ETS proteins in the tumorigenesis of epithelioid sarcoma has yet to be elucidated.

In summary, epithelioid sarcomas can label with antibodies to the N-terminus of ERG, which may cause diagnostic confusion with angiosarcoma, particularly in cases that have a pseudovascular appearance. Therefore, a panel of antibodies including SMARCB1 and CD31, as well as careful selection of the ERG antibody is recommended in evaluation of epithelioid sarcomas and angiosarcomas.

\section{Comment}

During the revision of this manuscript, Miettinen et al reported a similar experience with ERG and D240 expression in epithelioid sarcomas. ${ }^{21}$ In their study, they found $38 \%$ of epithelioid sarcomas (41 out of 109) labeled with ERG using the same antibody to the N-terminus $(1: 100$, Clone 9FY, Biocare Medical). Seven out of nine cases (78\%) also had labeling for D2-40 (1:100, Convance/Signet, Princeton, NJ, USA). These findings are similar to our study; although a higher percentage of ERG labeling was seen in our study possibly due to technical differences or vagaries of sample size. Other vascular markers including CD31 (as in our study), claudin 5, and Prox 1 were found to be negative in epithelioid sarcoma. No rearrangement of $E R G$ was seen using fluorescence in-situ hybridization in 16 tested epithelioid sarcomas. FLI1 expression and Erg C-terminus antibody were not performed in their study. Both of our studies emphasize the use of an antibody panel in differentiating epithelioid sarcoma from angiosarcomas.

\section{Acknowledgements}

We thank Kim-Anh Vu for her assistance in creating the figures.

\section{Disclosure/conflict of interest}

The authors declare no conflict of interest.

\section{References}

1 McKay KM, Doyle LA, Lazar AJ, et al. Expression of ERG, an ETS family transcription factor, distinguishes cutaneous angiosarcoma from histological mimics. Histopathology 2012;61:989-991.

2 Folpe AL, Chand EM, Goldblum JR, et al. Expression of Fli-1, a nuclear transcription factor, distinguishes vascular neoplasms from potential mimics. Am J Surg Pathol 2001;25:1061-1066.

3 Miettinen M, Wang ZF, Paetau A, et al. ERG transcription factor as an immunohistochemical marker for vascular endothelial tumors and prostatic carcinoma. Am J Surg Pathol 2011;35:432-441.

4 Yaskiv O, Rubin BP, He H, et al. ERG protein expression in human tumors detected with a rabbit monoclonal antibody. Am J Clin Pathol 2012;138:803-810.

5 Sakharpe A, Lahat G, Gulamhusein T, et al. Epithelioid sarcoma and unclassified sarcoma with epithelioid features: clinicopathological variables, molecular markers, and a new experimental model. Oncologist 2011;16:512-522.

6 von Hochstetter AR, Grant JW, Meyer VE, et al. Angiomatoid variant of epithelioid sarcoma. The value of immunohistochemistry in the differential diagnosis. Chir Organi Mov 1990;75:158-162.

7 von Hochstetter AR, Meyer VE, Grant JW, et al. Epithelioid sarcoma mimicking angiosarcoma: the value of immunohistochemistry in the differential diagnosis. Virchows Arch A Pathol Anat Histopathol 1991;418:271-278.

8 Wick MR, Manivel JC. Epithelioid sarcoma and epithelioid hemangioendothelioma: an immunocytochemical and lectin-histochemical comparison. Virchows Arch A Pathol Anat Histopathol 1987;410:309-316.

9 Chase DR, Enzinger FM. Epithelioid sarcoma. Diagnosis, prognostic indicators, and treatment. Am J Surg Pathol 1985;9:241-263.

10 Mills SE, Fechner RE, Bruns DE, et al. Intermediate filaments in eosinophilic cells of epithelioid sarcoma: a light-microscopic, ultrastructural, and electrophoretic study. Am J Surg Pathol 1981;5:195-202.

11 Fletcher CD, Beham A, Bekir S, et al. Epithelioid angiosarcoma of deep soft tissue: a distinctive tumor readily mistaken for an epithelial neoplasm. Am J Surg Pathol 1991;15:915-924. 
12 Chase DR, Enzinger FM, Weiss SW, et al. Keratin in epithelioid sarcoma. An immunohistochemical study. Am J Surg Pathol 1984;8:435-441.

13 Arber DA, Kandalaft PL, Mehta P, et al. Vimentinnegative epithelioid sarcoma. The value of an immunohistochemical panel that includes CD34. Am J Surg Pathol 1993;17:302-307.

14 Hornick JL, Dal Cin P, Fletcher CD. Loss of SMARCB1 expression is characteristic of both conventional and proximal-type epithelioid sarcoma. Am J Surg Pathol 2009;33:542-550.

15 Hollmann TJ, Hornick JL. SMARCB1-deficient tumors: diagnostic features and molecular genetics. Am J Surg Pathol 2011;35:e47-e63.

16 Sullivan LM, Folpe AL, Pawel BR, et al. Epithelioid sarcoma is associated with a high percentage of SMARCB1 deletions. Mod Pathol 2013;26:385-392.
17 Arvand A, Denny CT. Biology of EWS/ETS fusions in Ewing's family tumors. Oncogene 2001;20: 5747-5754.

18 Lushnikova T, Knuutila S, Miettinen M. DNA copy number changes in epithelioid sarcoma and its variants: a comparative genomic hybridization study. Mod Pathol 2000;13:1092-1096.

19 Wang WL, Patel NR, Caragea M, et al. Expression of ERG, an ETS family transcription factor, identifies ERG-rearranged Ewing sarcoma. Mod Pathol 2012;25:1378-1383.

20 Martens JH. Acute myeloid leukemia: a central role for the ETS factor ERG. Int J Biochem Cell Biol 2011;43:1413-1416.

21 Miettinen M, Wang Z, Sarlomo-Rikala M, et al. ERG Expression in Epithelioid Sarcoma. Am J Surg Pathol 2013;37:1580-1585. 